\title{
SEARCHES FOR CHARGED HIGGS BOSONS
}

\author{
MATTIAS ELLERT \\ Department of Radiation Sciences, Uppsala University \\ Box 535, SE-751 21 Uppsala, Sweden \\ E-mail: mattias.ellert@tsl.uu.se
}

\begin{abstract}
The results of the searches for charged Higgs bosons at the four experiments at the LEP collider have been combined in order derive an exclusion limit using the largest possible data sample. This combined analysis excludes the existence of charged Higgs bosons with masses lower than $78.6 \mathrm{GeV} / c^{2}$ at $95 \%$ confidence level.
\end{abstract}

\section{Introduction}

Charged Higgs bosons are predicted to exist in several extensions to the Standard Model. The simplest of these extensions is the so called two Higgs doublet model (2HDM), of which the minimal supersymmetric model (MSSM) is a special case where additional constraints on the model parameters originating from supersymmetry have been imposed.

In the MSSM these additional constraints restrict the tree-level mass of the charged Higgs boson to be greater than that of the $\mathrm{W}^{ \pm}$boson. Some choices of the MSSM model parameters can however result in loop corrections that give a lower mass.

\section{Searches at LEP}

In an electron positron collider like LEP the production of charged Higgs bosons occurs mainly through pair production $\left(e^{+} e^{-} \longrightarrow \gamma / \mathrm{Z}^{0} \longrightarrow \mathrm{H}^{+} \mathrm{H}^{-}\right)$. In the $2 \mathrm{HDM}$ the tree-level cross-section for this process can be calculated as a function of a single parameter - the mass of the charged Higgs boson.

The couplings between the charged Higgs boson and other particles are proportional to the masses of the particles. This means that charged Higgs bosons decay into the heaviest particles kinematically allowed. At the LEP collider this means either a $c s$ quark pair or a $\tau \nu_{\tau}$ lepton pair. The branching ratio between the two possible decays depends on the parameters of the model.

Since the charged Higgs bosons are pair produced, this results in three possible final states: a hadronic final state $(c \bar{s} \bar{c} s)$, a leptonic final state $\left(\tau^{+} \nu_{\tau} \tau^{-} \bar{\nu}_{\tau}\right)$ and a semi-leptonic final state $\left(c \bar{s} \tau^{-} \bar{\nu}_{\tau}\right.$ or $\left.\tau^{+} \nu_{\tau} \bar{c} s\right)$.

The data used in the analysis were collected by the four experiments at the LEP collider during 1998-2000 at several different centre of mass energies

SUSYproc: submitted to World Scientific on July 30, 2001 
ranging from 189 to $208 \mathrm{GeV}$. In the analysis each final state at each centre of mass energy at each experiment was treated as a separate channel.

The mass limits obtained by the experiments at the LEP collider are given in Table 1. In addition to the observed limit, an expected limit obtained by taking the median of the limits derived from a large number of Monte Carlo generated results compatible with the background expectation is given. The large deviations between the observed and expected limits from the L3 experiment are due to an excess of observed events with a reconstructed mass around $68 \mathrm{GeV} / c^{2}$. This excess is not confirmed by the other three experiments. The observed and expected exclusion limits as a function of $\mathrm{BR}\left(\mathrm{H}^{+} \longrightarrow \tau^{+} \nu_{\tau}\right)$ are shown in Figure 1. The combined analysis excludes the existence of charged Higgs bosons with masses lower than $78.6 \mathrm{GeV} / c^{2}$ at $95 \%$ confidence level for any branching ratio.

Table 1. Observed and expected $95 \%$ confidence level mass limits $\left(\mathrm{GeV} / c^{2}\right)$ for charged Higgs bosons obtained by the four experiments at the LEP collider. Limits are given for $\mathrm{BR}\left(\mathrm{H}^{+} \longrightarrow \tau^{+} \nu_{\tau}\right)=0$ and 1 , as well as independently of the branching ratio.

\begin{tabular}{|c|c|c|c|c|c|c|}
\hline \multirow{2}{*}{ Experiment } & \multicolumn{2}{|c|}{$\overline{\mathrm{BR}}=0$} & \multicolumn{2}{|c|}{$\mathrm{BR}=1$} & \multicolumn{2}{|c|}{ any BR } \\
\hline & obs. & exp. & obs. & exp. & obs. & exp. \\
\hline$\overline{\text { ALEPI }}$ & 80.7 & 78.1 & 83.4 & 86.9 & 78.0 & 76.9 \\
\hline DELPHI $^{2}$ & 77.4 & 77.0 & 85.4 & 89.3 & 73.8 & 75.4 \\
\hline $\mathrm{L}^{3}$ & 67.7 & 76.5 & 82.8 & 84.7 & 65.6 & 75.1 \\
\hline $\mathrm{OPAL}^{4}$ & 76.2 & 77.1 & 84.5 & 86.5 & 72.2 & 74.5 \\
\hline combined $^{5}$ & 81.0 & 80.2 & 89.6 & 92.1 & 78.6 & 78.8 \\
\hline
\end{tabular}

\section{Acknowledgements}

I would like to thank the LEP collaborations and the LEP Working Group for Higgs Boson Searches for providing the results presented here.

\section{References}

1. ALEPH collaboration, Search for charged Higgs bosons in $e^{+} e^{-}$collisions at energies up to $\sqrt{s}=209 \mathrm{GeV}$, ALEPH/2001-016 CONF/2001-013.

2. DELPHI collaboration, Search for Charged Higgs Bosons in $e^{+} e^{-}$Collisions at LEP, DELPHI 2001-071 CONF 499.

3. L3 collaboration, Search for Charged Higgs Bosons in $e^{+} e^{-}$Collisions at Centre-of-Mass Energies up to 209 GeV, L3 note 2686 (2001). 


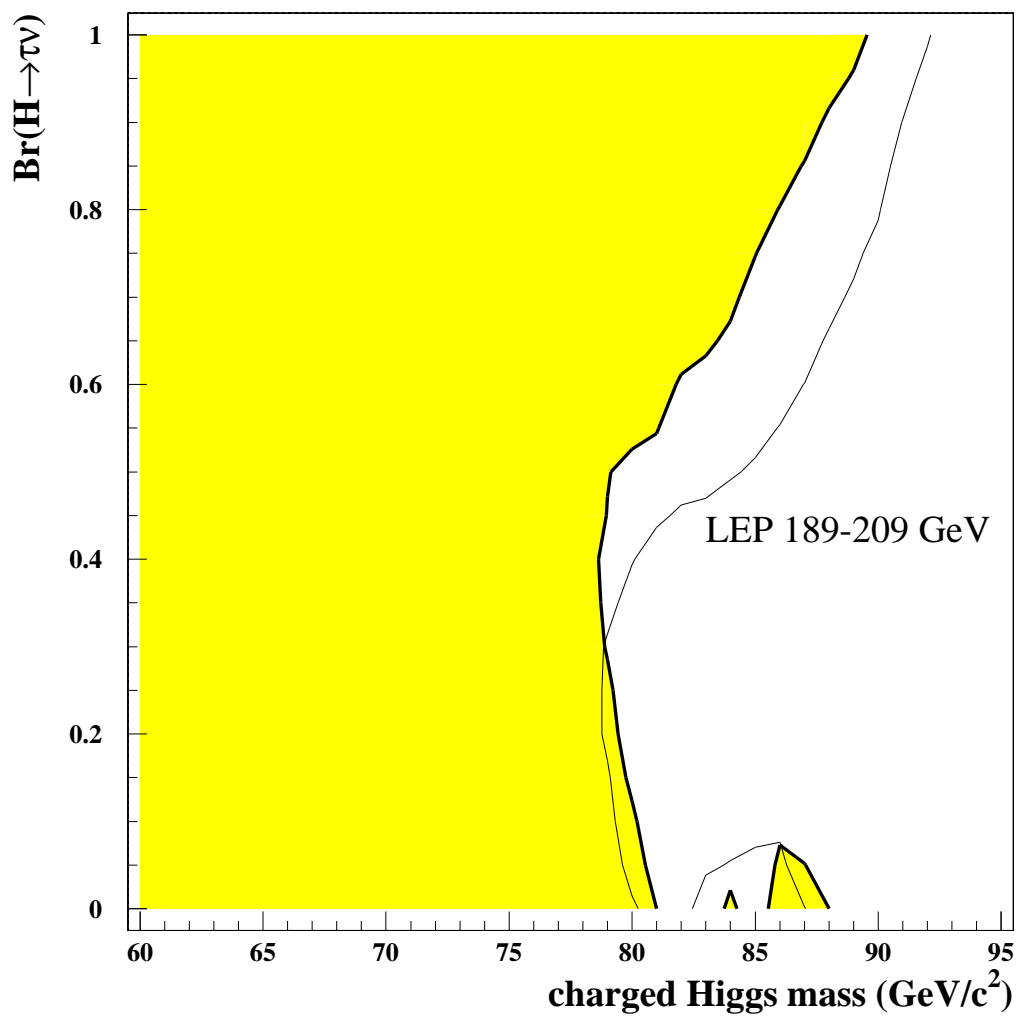

Figure 1. The 95\% confidence level mass limit for charged Higgs bosons as a function of $\mathrm{BR}\left(\mathrm{H}^{+} \longrightarrow \tau^{+} \nu_{\tau}\right)$ obtained when combining the results from the searches performed at the four LEP experiments. The observed exclusion limit is indicated by the thick solid line and the expected exclusion limit by the thin solid line.

4. OPAL collaboration, Searches for Higgs Bosons in Extensions to the Standard Model in $e^{+} e^{-}$Collisions at the Highest LEP Energies, OPAL PN 472 (2001).

5. The LEP Working Group for Higgs Boson Searches, Search for Charged Higgs Bosons: Preliminary Combined Results using LEP Data Collected at Energies up to $209 \mathrm{GeV}$, LHWG note 2001-05. 